\section{Feuilleton. \\ Eine Expedition zur Erforschung der physiologischen Wirkungen des Hochgebirges.}

II.

Von Dr. W. Caspari, Assistenten am thierphysiologisclen Institut der Königlichen Landwirthschaftlichen Hochschule.

In No. 50 des vorigen Jahrganges dieser Wochenschrift hat Professor Loewy die Vorbereitung der Expedition beschrieben, welche von den dort genannten Herren unternommen wurde, um die Einwirkung des Hochgebirges auf den Organismus zu studiren. Mir ist die Aufgabe geworden, in kurzen Zügen den Verla uf des Unternehmens zu schildern und darzuthun, in welcher Weise die so vorbereiteten Versuche zur Ausführung gelangten. Auch ich bin keineswegs in der Lage, zahlenmässige Resultate mitzutheilen. Die Feststellung derselben wird noch längere Zeit in Anspruch nehmen. Doch mag es für den ärztlichen Leser immerhin nicht ohne Interesse sein, etwas Näheres über den Gang dieser Untersuchungen zu erfahren, auch wenn noch nicht alle Früchte derselben gereift sind.

Die zahlreichen grossen Kisten waren längst nach Brienz vorausgeschickt. Einzelne besonders zerbrechliche Apparate sollten jedoch als Handgepäck mitgehen. Am 2. August trafen wir uns in Basel und setzten unsere Reise gemeinsam fort. In Bern überzeugten wir uns von dem Wohlbefinden des einen Theiles unserer Hunde, welchen Herr Professor Kronecker in liebenswürdiger Weise in seinem Institut Gastfreundschaft gewährt hatte; dann fuhren wir nach Brienz, wo wir am 4. August anlangten.

Der Tag ging hin mit dem Einrichten unseres Laboratoriums. Die Räume, welche uns für unsere Versuche zur Verfügung standen, müssen geradezu als ideal für diese Zwecke bezeichnet werden. Unsere Wohnräume lagen in der ersten Etage des Hauses: drei geräumige Schlafzimmer, ein freundliches Wohnzimmer, Küche und Schlafzimmer für unsere mitgenommene Köchin. So fehlte es uns hier nicht an dem Comfort des Lebens, ein Umstand, der für solche Selbstversuche von grösster Wichtigkeit ist. Aber auch für den Comfort der Arbeit war über Erwarten gesorgt. Das Laboratorium richteten wir in der sonst unbewohnten zweiten Etage des Hauses ein, so dass weder wir noch die Hausbewohner durch unsere Thätigkeit und die unvermeidlichen Insulte auf die Geruchsnerven allzu sehr belästigt wurden. Hier stand uns zunächst eine Küche zur Verfügung, deren Heerd als Digestorium vorzügliche Dienste leistete. Der Boden war mit Steinfliesen belegt, was für die Reinhaltung des Raumes von nicht zu unterschätzender Bedeutung war. Ausserdem war Wasserleitung vorhanden mit Wasserausguss und einem vorzüglichen Abwaschtisch. Diese Küche diente als chemischer Arbeitsraum. Ferner wurde an das Fenster ein Tisch gerückt, welcher für die mikroskopischen Untersuchungen sowie Dichtebestimmung von Blut und Serum benutzt wurde. Neben der Küche lag ein zweites Zimmer, in dem wir unsere grosse Waage sowie den Zuntz'schen Gasanalysenapparat, den wir complet aus Berlin mitgebracht hatten, aufstellten. Ausserdem konnten wir noch ein grosses leeres Zimmer zum Aufbewahren unserer Vorräthe und Utensilien benutzen.

Nachdem wir uns häuslich eingerichtet hatten, begannen wir am 5. August den Stoffwechselversuch durch Abgrenzen des Kothes. Wir hatten aus unseren Vorversuchen die Erkenntniss gewonnen, dass die gebräuchlichen Abgrenzungsmittel beim Versuche am Menschen, wenn auch in individuell sehr verschiedenem Maasse, insgesammt ihre Bedenken haben. Selbst die bei Hundeversuchen so gut bewährte Kieselsäure hatte völlig versagt. Es ersćhien uns danach am exaktesten, den Koth der einzelnen Perioden durch Klystire abzugrenzen, die wir solange fortsetzten, bis die Reste eines am Tage vorher genossenen Gemüses - besonders Karollen hoben sich gut ab - in Kothe erschienen.

Die Gesammteintheilung des Tages war während des Brienzer Aufenthaltes folgende: Morgens vor $6 \mathrm{Uhr}$ wurde im Bette Puls gezählt und Temperatur gemessen, sodann der Harn abgegrenzt. Der Morgenharn wurde zu dem des vorhergehenden Tages hinzugefügt. Dann begaben wir uns in das Laboratorium hinauf, wo das Nacktgewicht nüchtern festgestellt sowie der Harn des verflossenen Tages gewogen und sein spezifisches Gewicht bestimmt wurde: Dann machten wir Toilette, nahmen unsere $200 \mathrm{ccm}$ Kaffee und wogen die Tagesration, bestehend in Cakes, Zucker, Butter, Käse, Marmelade, Schokolade ab. An anderen Tagen aber begab sich der Eine oder Andere nach Feststellung des Körpergewichts und der Abgrenzung des Harnes wieder zu Bett. Dort wurden an ihm Respirationsversuche gemacht, um die Grösse seines Sauerstoffverbrauches nüchtern bei vollkommener Ruhe zu ermitteln. Dieser Werth bildet die Grundlage für die Feststellung des Mehrverbrauches unter verschiedenen Versuchsbedingungen.

Der Vormittag verging unter verschiedener Thätigkeit. Der Eine analysirte die am Morgen oder Tags zuvor gewonnenen Athemproben, Andere waren mit Blutuntersuchungen, wieder Andere mit meteorologischen Beobachtungen beschäftigt.

Um 21/2 Uhr wurde meist zu Mittag gegessen. Das Mittagsmahl bestand in zwei Beefsteaks aus sterilisirtem Fleisch, täglich gleichen Mengen Reis und abwechselnd Schoten, Karotten oder Spinat in äquivalenten Mengen. Dazu ein kleines Milchbrod. um die Portionen quantitativ ausessen zu können. Sonst wurde von Brod in der Nahrung völlig abgesehen, da dessen Zusammensetzung zu ungleichmässig ist. Von der täglichen Brödchensendung wurde stets ein Exemplar zur Analyse aufgehoben.

An Getränken nahmen wir im Laufe des Tages Wasser, gelegentlich mit etwas Citronensäure, sowie Thee und Kaffee zu uns. Professor Löwy und ich tranken auch 1-2 Flaschen Bier, selbstverständlich analysirt! Die übrigen vier Herren enthielten sich völlig des Alkohols. Auch die Getränke wurden, ebenso wie die Speisen, Harn und Koth, genau gewogen. Auf diese Weise hatten wir unter Berücksichtigung des Körpergewichts alle Daten für die Berechnung der insensiblen Perspiration, d. h. der von Haut und Lungen während des Tages abgegebenen Stoffmenge. ${ }^{\text {) }}$ )

Der Nachmittag wurde in derselben Weise wie der Vormittag mit Arbeiten verschiedener Art verbracht. So verstrich die Zeit schnell, bis die Abendmahlzeit uns wieder zu gemeinsamem Schmause versammelte. Der Abend war den nothwendigen Notizen und der Korrespondenz gewidmet, und so war es trotz unserer guten Absicht, früh zu Bette zu gehen, stets etwa 1/211 Uhr, ehe wir das Lager aufsuchen konnten. Pulszählung und Temperaturmessung im Bett beschloss das Tagewerk.

Der gesammte Gang der Untersuchungen während des Aufenthaltes in der Brienzer Gegend war kurz folgender. In den ersten sechs Tagen wurde der Stoffwechsel bei Ruhe und mässiger Arbeit im Laboratorium festgestellt, vom vierten Tage ab auch einige Daten gewonnen für den Sauerstoffverbrauch bei Steigarbeit, wobei jedoch weitere Märsche vermieden wurden. Es wurde dann eine Trennung der Expedition in zwei verschiedene Gruppen vorgenommen. Die eine derselben, unter Führung von Löwy, begab sich direkt auf den Gipfel des Brienzer Rothorns (cirka $2300 \mathrm{~m}$ ), um so die Veränderungen im Haushalte des Organismus bei unmittelbarem Uebergange von der Ebene zu einer mittleren Berghöhe zu studiren. Von den Herren dieser Gruppe waren zwei, Löwy und Müller, zwar schon wiederholte Male auf bedeutenderen Höhen gewesen, momentan aber völlig untrainirt. Der dritte dagegen, Kolmer, welcher während des Semesters in Bern gewesen war, hatte die Zeit benutzt, um seinen ohnedies schon aussergewöhnlich kräftigen Körper durch grosse Hochtouren für die beabsichtigten Versuche vorzubereiten. So durften wir hoffen, nicht nur interessante Vergleichsresultate zwischen dieser Gruppe und den im Thal gebliebenen Theilnehmern der Expedition zu erlangen, sondern auch einen gewissen Unterschied zwischen dem trainirten Mitgliede und den untrainirten der oberen Gruppe festzustellen. Nachdem die Herren hinlänglich lange auf der Höhe geweilt hatten, um den Verbrauch bei Ruhe festzustellen, begann der zweite Abschnitt der Versuche,

1) Vergl. Löwy, Beiträge zum Stoff- und Energieumsatz des Menschen. Archiv für Anatomie und Physiologie, Physiologische Abtheilung 1901, S. 319. 
in welchem voll beiden T'lıeilen der Expedition Märsche voll mittlerer Ausdehnung geleistet wurden, welche stets mit Untersucliung der Athmung verbunden waren. $\mathrm{Zu}$ diesem Zwecke stieg die Thalgruppe bis etwa zur Hälfte des Berges (560-1300 m) hinauf, während die obere Gruppe mittels der Bahll etwa den halben Berg hinuiterfuhr, um dann ihrerseits die obere Hälfte zum Aufstiege zu benutzell. Allmählich wurden die Marschleistungen gesteigert, bis endlich die „Periode der grossen Märsche" begann. An diesen Tagen erreichte die Berggruppe mit Benutzung der Bahn den Fuss des Rothorns, um von dort gemeinsam mit der Thalgruppe den ganzen Berg bis zum Gipfel zu erklimmen.

Am 18. Versuchstage folgte dann ein neuer Abschnitt. Die Berggruppe kehrte ins Thal zurück, während diejenigen Theilnehmer der Expedition, welche die Wohnung im Thale beibelialten und sich dann durch die vorhergehenden Märsche auf das zweckmässigste für den Aufenthalt in der Höhe allmählich trainirt hatten, nunmehr ihren dauernden Aufenthalt auf dem Gipfel des Rothorn nahmen. Nach weiteren sechs Tagen vereinigten sich säınmtliclıe Theilnehılıer wieder in Brienz, unı nunmehr die Fahrt zum Monte Rosa allzutreten.

Ausser den rein physiologischen Beobachtungen wurden auch Feuchtigkeit, Druck, Temperatur der Luft, Elektrizitätszerstreuung in der Atmosphäre, sowie elektrisches Potentialgefälle stets auf beiden Stationen untersucht. Schliesslich wurden auch einige Versuche gemacht, den Schweiss während eines Marsches zu sammeln. Denn wie die Ver'suche von Argutinsky ${ }^{1}$ ), $\mathrm{Kramel}^{2}{ }^{2}$ ), Zuntz und Schumburgis), sowie die von Löwy und Müller ${ }^{4}$ ) gezeigt haben, ist die durch den Schweiss während eines Marsches besonders an heissen Sommertagen ausgeschiedene Stickstoffmenge durchaus nicht unbedeutend. Um nun während eines Marsches den Schweiss zu sammeln, verfuhren wir folgendermaassen: Eine geeignete wollene Unterkleidung wurde vor den Versuche sorgfältig mit essigsaurem Wasser ausgelaugt. Hierauf wurden die Kleider getrocknet und an Versuchstage vor denı Marsche angelegt, nachdem der gesammte Körper der Versuchsperson vorher sorgfältig mit warmem Wasser abgewasclien wolden war. Nach der Rückkehr vom Marscle wurde der Körper von neuem gründlich abgewaschen und die Kleider wiederum ausgelaugt. Körper- und Kleiderwaschwasser wurde dann vereinigt, auf ein geringeres Volumen eingedampft und zur Analyse nach Berlin gesandt.

Dem allgemeinen Arbeitsplan gemäss begannen wir am 9. August die Messung des Gaswechsels beim Bergsteigen. Wir hatten einen Weg von etwa $1 / 2$ Stunde zurückzulegen, bis wir den "Start" erreichten. Es war dies der erste Kilometerstein auf der Bahntrace der Brienzer Rotlıornbahn. Die Trace der Rothornbahn wählten wir naclı dem Vorgange von Bürgi ${ }^{5}$ ). Sie eignet sich besonders gut für solche Zwecke, weil sie fast in ihrem ganzen Verlauf eille gleichmässige Steigung von $25 \%$ besitzt und, da auch die Wegstrecken genau gemessen sind, eine leichte Berechnung der bein Steigen geleisteten Arbeit gestattet. Wir alle silld der Direktion der Rothornbahn, insbesondere Herrn Direktor Hochstrasser, für ihr liebenswürdiges Entgegenkommen zu aufrichtigstem Danke verpflichtet.

Beim Bergaufsteigen trug das Versuchsobjekt die trockene Gasuhr, an welcher die Menge der durchstreifenden Athemluft abgelesen werden konnte, auf dem Rücken. Um diese unvermeidliche Last möglichst den Verhältnissen beim Bergsteigen anzupassen, war die Gasuhr auf einer „Kraxe“ befestigt, wie sie die Alpenbewohıer beim Tragen von Lasten benutzen. Diese Einrichtung liat sich vortrefflich bewährt. Die Gasuhr war in üblicher Weise mit einer Röhre zum Sammeln einer Durclischnittsprobe der Athemluft armirt. Die Versuche zerfielen in eine Vorperiode, während welcher sich die Athmung auf die der Arbeit entsprechende Grösse einstellte, und die eigentliche Versuchsperiode, in welcher eine Durchschnittsprobe des Gases aufgefangen wurde. Der ganze Versuch dauerte nur etwa fünf Mi-

1) Ueber die Stickstoffausscheidung durch den Schweiss. Pflü g'e r's Archiv für Hygiene Bd. XLVI, S. כ94.

$\left.{ }^{2}\right)$ Ueber cie Beziehung der Kleidung; zur Hautthätigkeit. Archiv für Hygiene Bd. X, S. 231.

3) Physiologie des Marsches. Bibliothek von Coler S. $198 \mathrm{ff}$.

4) A. Löw y, a. a. O. S. 299.

5) Der respiratorische Gaswechsel bei Ruhe und Arbeit auf den Bergen. Archiv für Anatomie und Physiologie, Physiologische Abtheilung 1900 , S. 501 . nuten. Waihrend des ganzen Marsches trug einer voll uns auf dem Haupte ein kleines Anemometer. Dieses war auf einer Art von kleinem Heln aufgeschraubt und bildete so eine äusserst phantastische Kopfbedeckung, die in den glühenden Sonnenstrahlen auf der ganz schattenlosen Balntrace besonder's schön glitzerte und blinkte. Der Zweck dieser Einrichtung war, den Luftzug festzustellen, dem die Versuchspersonen ausgesetzt warell, da die Kenntnis desselben wichtig ist für das Verständniss der Wärmeregulation der Marschirenden.

Es braucht wohl kaum erwähnt zu werden, dass die Bewohner und Sommergäste von Brienz sich über den Zweck unseres Aufentlıaltes weidlich die Köpfe zerbrachen und die abenteuerlichsten Gerüclite über uns und unsel seltsames Treiben kursirten, und dies um so mehr, weil wir, um nicht gestört $\mathrm{zu}$ werden, uns in einen dichten Schleier geheimnissvollen Stillschweigens hüllten.

Das grösste Aufsehen erregten jedoch einige Versuche, welche wir machten, um einen ungefähren Einblick in den Sauerstoffverbrauch beim Schwimmen zu gewinnen. Es sah auch sonderbar genug aus, wein Helr Kolmer, welcher allein als Versuchsobjekt in Betracht kam, hinter dem langsam volwärts gerudertell Boote einherschwamm. Im Boote stand die Gasuhr, und der Mund des Schwimmers war in der üblichen Weise mit derselben verbunden, nur dass der Gummischlauch, welcher sonst möglichst kurz gewählt wird, eine Länge von sieben Metern besass und so gleich der berühmten Seeschlange in grossen Windungen auf dem Wasser hinglitt. Die Inspirationsluft musste natürlich aus der freien Atmosphäre aufgenommen werden. Um dies zu erreichen, trug Herr Kolmer auf seinem Haupte den oben erwähnten Helm, welcher sonst zur Befestigung des Anemometers diente. Jetzt ragte von seiner Spitze das freie Ende des Inspirationsschlauches als Schornstein ill die Luft. Vergegenwärtigt man sich noch, dass die Nase des Schwimmenden durch die übliche Klemıne verschlossen war, so wird man zugeben müssen, dass das Staunen des Publikums nicht ganz ohne Berechtigung war. Wir haben nur wenige bezügliche Versuche machen können; deun die erste Versuchsperiode war nur kurz, und später waren wir durch unsere anderweitige Thatigkeit, besonders die Marscharbeit, völlig in Anspruch genommen. Wir können daher auch nicht erwarten, dass diese Schwimnversuche mehr als eine Orientirung liefern werden. Immerhin verdient die Thatsache, dass beim Schwimnien der Gaswechsel ganz überraschend hohe Werthe zeigt, einiges Interesse.

(Schluss folgt.) 